\title{
The Quick PsychoDiagnostics Panel was accurate for identifying psychiatric disorders in primary care
}

Shedler J, Beck A, Bensen S. Practical mental health assessment in primary care. Validity and utility of the Quick

PsychoDiagnostics Panel.J Fam Pract 2000 Jul;49:614-21. QUESTION: Is the Quick PsychoDiagnostics (QPD) Panel an accurate assessment tool
for identifying psychiatric disorders in primary care?

Design

A blinded comparison of the QPD Panel with Structured Clinical Interview for DSM-IV (SCID) diagnoses for major depression (MD), generalised anxiety disorder (GAD), panic disorder, and obsessive compulsive disorder (OCD) (study 1); and a blinded comparison of the QPD Panel with clinician diagnoses of alcohol or substance abuse (study 2).

\section{Setting}

Health maintenance organisations (HMOs) in Colorado, USA

\section{Patients}

Study 1: 203 HMO patients (mean age $41 \mathrm{y},>66 \%$ women) who were referred by their physicians or self referred for a first time mental health consultation and were not receiving mental health treatment at study entry. Study 2: 159 patients (mean age 42 y, $71 \%$ women) enrolled in an HMO health plan. $46(29 \%)$ of these patients had been diagnosed by their physicians as having alcohol or substance abuse and were referred to a chemical dependency clinic for treatment.

Sources of funding: Research and Development fund of Kaiser Permanente,

Colorado Region and SmithKline Beecham.

For correspondence: Dr J Shedler, Digital Diagnostics, Inc, 225 North Mill Street, Suite 207, Aspen, CO

81611, USA.Fax +1 9705443882.

\section{Description of test and diagnostic standards}

The QPD Panel is an automated test that patients completed on handheld computers by answering true/false questions. The QPD Panel screened for 9 DSM-IV mental disorders. A diagnostic report was printed immediately. The diagnostic standard was SCID diagnoses in study 1 and clinician diagnoses in study 2 .

\section{Main outcome measures}

Sensitivity, specificity, and likelihood ratios.

\section{Main results}

The table shows the results for studies 1 and 2 .

\section{Conclusions}

The Quick PsychoDiagnostics (QPD) Panel had high sensitivity and specificity for detecting depression and alcohol or substance abuse in primary care. It had moderate sensitivity and high specificity for detecting generalised anxiety, panic, and obsessive compulsive disorders.

\section{COMMENTARY}

Many studies point to the high prevalence of psychiatric disorders in primary care and the variable ability of primary care physicians to detect these. ${ }^{1}$ Screening for these disorders is, in principle, desirable but not widely applied. ${ }^{2}$ Screening questionnaires such as the General Health Questionnaire (GHQ) have acceptable validity coefficients and provide an overall screen for common psychological disorders. ${ }^{3}$ Some of these common disorders may be DSM disorders, but many are subthreshold states. Two features of the OPD Panel are that it screens for a range of disorders commonly seen in primary care, including substance abuse, and it can be given in an automated form.

Shedler $e t a l$ s results are encouraging. The limitations of the study are discussed in the paper, but of particular importance is the use of a mental health sample rather than a general primary care sample, thus restricting generalisability.

The QPD Panel may be useful for alerting physicians to the possibility of psychological disorder and its subtypes, auditing patients about their unmet needs, early detection and treatment, assessing change, and assisting in teaching.

The results suggest that the OPD Panel is worthy of further assessment. In particular, its practical value requires assessment in a general primary care sample and across various primary care settings in different countries because what is useful and acceptable in one setting may not be in another. How does it compare to the use of questionnairesfor example, the GHQ? Does it have a research use? The use of a screening device does not replace sound clinical practice, and it is known that the ability of primary care physicians to detect psychological problems in patients is related to their interview skills. ${ }^{1}$ How can the QPD Panel be best used in clinical practice and, more importantly, does its use have any effect on patient outcome?

Jed Boardman, MBBS, FRCPsych, PhD Guy's, King's E于 St Thomas'Hospitals Medical School King's College London, UK

1 Goldberg DP, Huxley P. Common mental disorders: a biosocial model. London: Tavistock/Routledge, 1992.

2 Attkisson CC,Zich JM. Depression in primary care:screening and detection. London: Routledge, 1990.

3 Goldberg D, Williams P. A user's guide to the general health questionnaire. Basingstoke: NFER-Nelson, 1988.

*SCID=Structured Clinical Interview for DSM-IV. Diagnostic terms defined in glossary; LRs calculated from data in article. 